\title{
DEVELOPMENT OF A COTS MASS STORAGE UNIT FOR THE SPACE READINESS COHERENT LIDAR EXPERIMENT
}

\author{
Karl Liggin, Computer and Data Systems Group, Avionics Laboratory, \\ NASA Marshall Space Flight Center Huntsville, Alabama \\ Porter Clark, Computer and Data Systems Group, Avionics Laboratory, \\ NASA Marshall Space Flight Center Huntsville, Alabama
}

\section{Background}

The technology to develop a Mass Storage Unit (MSU) using commercial-off-theshelf (COTS) hard drives is an on-going challenge to meet the Space Readiness Coherent Lidar Experiment (SPARCLE) program requirements. A conceptual view of SPARCLE's laser collecting atmospheric data from the shuttle is shown in Figure 1. The determination to develop this technology required several in depth studies before an actual COTS hard drive was selected to continue this effort.

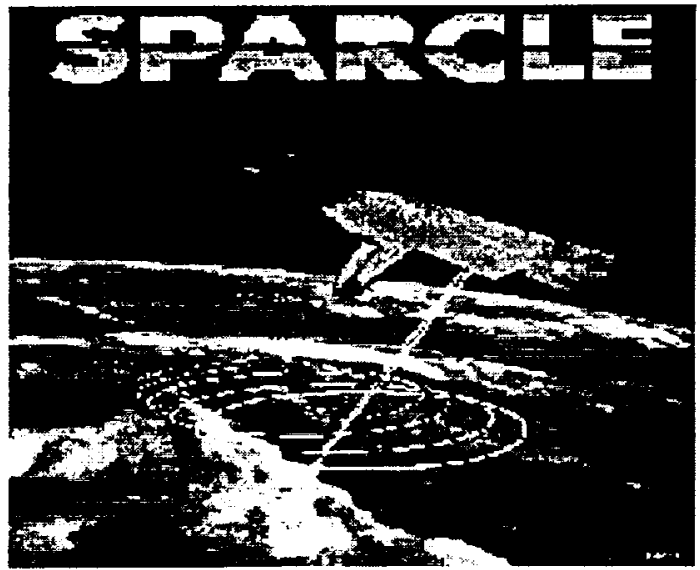

Figure 1. SPARCLE collecting data from the shuttle.

However, because this COTS technology is unproven, the question still remains. How far could NASA go faster, cheaper, and better, if commercial-off-the-shelf hard drives could fly?

Continuing the development of the MSU can, and will, serve future NASA programs that require larger data storage and more on-board processing. This effort will provide a greater means to other programs that have limited downlink capabilities within their payload telemetry streams. Due to the program cancellation this paper will only contain preliminary and theoretical results.

The low cost approach was driven by the program to develop and demonstrate new technologies "Faster, Cheaper, and Better (FCB)" under the guidelines of the New Millennium Program (NMP). Therefore, this was one of the main driving factors to select, test, and qualify a COTS magnetic disk drive. The other factors were driven by the requirements of the SPARCLE program to develop a laser, space-based Doppler system that could demonstrate the global measurement of atmospheric wind velocities for the Global Hydrology and Climate Center (GHCC).

\section{System Requirements}

The goal of the SPARCLE mission was to fabricate a coherent Doppler wind lidar system that would be designed to fly on the Space Shuttle within the Hitchhiker cans, and would measure the tropospheric line-of-sight and the horizontal vector wind profiles as a function of altitude.

SPARCLE PAMLAD CONIICLRATION CONCEPI

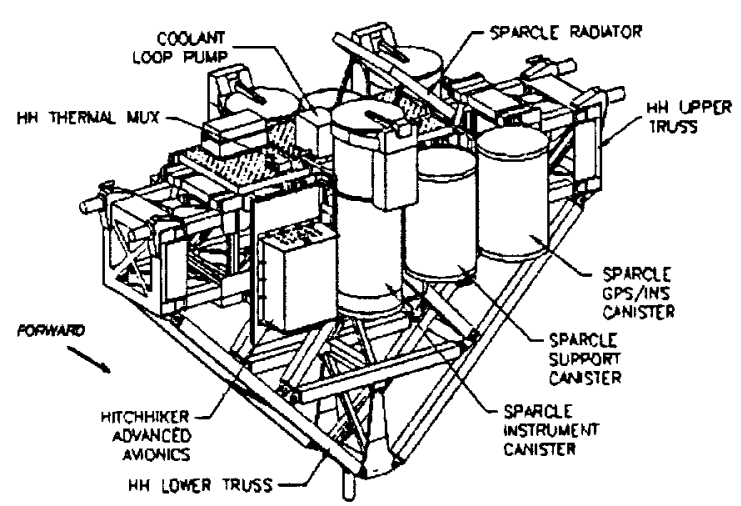

Figure 2. Hitchhiker Gas Cans 
This system would require a Command and Data Management System (CDMS) that would consist of Data Acquisition Control Unit and a Mass Storage Unit (MSU) that could collect, measure and store the data processed from the atmospheric return signal. The Electronics Receiver Unit (ERU) would control the firing signal to the Lidar Transceiver Unit (LTU) that would control the laser. It would also control signal processing of the reference detector $/$ preamplifier of the laser's master and slave oscillator.

The requirement calls for the ERU to capture the atmospheric signal 1.2 times the Nyquist frequency of the Intermediate Frequency (IF) bandwidth, where the IF bandwidth is greater than or equal to $335 \mathrm{MHz}$. Since the laser has a requirement to fire at a rate 6 times per second, the selected A/Ds for the ERU were required to have a sampling rate of 500 million samples per second to capture the atmospheric signal that contained the necessary data. The mass storage capacity required is given by following equations below:

\section{Given:}

- IF bandwidth $\geq 335 \mathrm{MHz}$

- Nyquist frequency $\left(f_{m y}\right)=167.5 \mathrm{MHZ}$

Where: $\quad f_{m y 4}=f_{S} / 2$

- Sampling frequency $\left(f_{.}\right)$

- $f_{S} \geq(2)(1.2)(167.5 \mathrm{MHz})$

- $\quad \geq 402 \mathrm{MHz}$

Therefore:

Storage Capacity (SC)

- $S C=\left(164 \mu \mathrm{s}^{*} 500 \mathrm{MB} / \mathrm{s}^{*} 2\right)+2.576 \mathrm{~KB}$

- $S C=166.6 \mathrm{MB}$ per scan

- $S C=166.6 \mathrm{MB}(6 \mathrm{~Hz})(3600 \mathrm{sec} / \mathrm{hr})(50 \mathrm{hr})$

- $S C=179.9 \mathrm{~GB} / 50$ hours

Where:

- A/D Sampling Rate $\quad=500 \mathrm{MHz}$

- Ancillary Data $=2.576 \mathrm{~KB}$

- Sampling Timeframe $=164 \mu \mathrm{sec}$

- Number of Channels $=2$

- Scan Rate $\quad=6 \mathrm{~Hz}$

- Total Timeframe = SOhrs.

Based on the calculations, the program scientist concluded that the capacity requirement for the
MSU only required $142 \mathrm{~GB}$ out of the $179.9 \mathrm{~GB}$ calculated, to meet the objectives of this experiment.

The initial CDMS budget requirements for the power, weight, and size (PWS) was passed along from Hitchhiker requirements, which limited the hard drives' selection process. The total PWS budget for the CDMS was given as not to exceed 125 watts of power, 35 pounds in weight, and to fit within an area of 8.8"x13.9"x13.9." The thermal requirements were defined as:

1) $-20^{\circ} \mathrm{C}$ to $40^{\circ} \mathrm{C}$ for the operating temperature range.

2) $-40^{\circ} \mathrm{C}$ to $60^{\circ} \mathrm{C}$ for the non-operational and survival temperature range.

The vibration requirement from Hitchhiker's Customer Accommodations and Requirements Specifications (CARS) document was the only other requirement that was imposed on the MSU selection process. The following requirement of the random vibration criteria was derived from CARS for all Hitchhiker payloads:

\section{All Axes}

- $20 \mathrm{~Hz}$

- $20-50 \mathrm{~Hz}$

(@) $\quad 0.01 \mathrm{~g}^{2} / \mathrm{Hz}$

- $50-600 \mathrm{~Hz}$

(a) $\quad+4.5 \mathrm{~dB} / \mathrm{oct}$

- $600-2000 \mathrm{~Hz}$

@

$0.040 \mathrm{~g}^{2} / \mathrm{Hz}$

- $2000 \mathrm{~Hz}$

- Composite

@

$-3.5 \mathrm{~dB} / \mathrm{oct}$

$0.01 \mathrm{~g}^{2} / \mathrm{Hz}$

Note:

1) Test duration is one minute per axis.

\section{Development and Design}

The Computer and Data System, Digital Development Design Team performed a study to determine if there were any available ruggedized COTS mass storage devices that would meet the requirements and the CDMS budget set by the SPARCLE program. The most available rugged mass storage devices came in the form factor of solid state memory, which was outside the cost range for this 
program. Therefore, the study began to move in the direction of magnetic disk drive.

Initially, the major concern with magnetic disk drive was their susceptibility to vibration, which is a major concern with the shuttle' launches. Then the study had to move in the direction of a COTS company that could provide some vibration data from any test that they had performed.

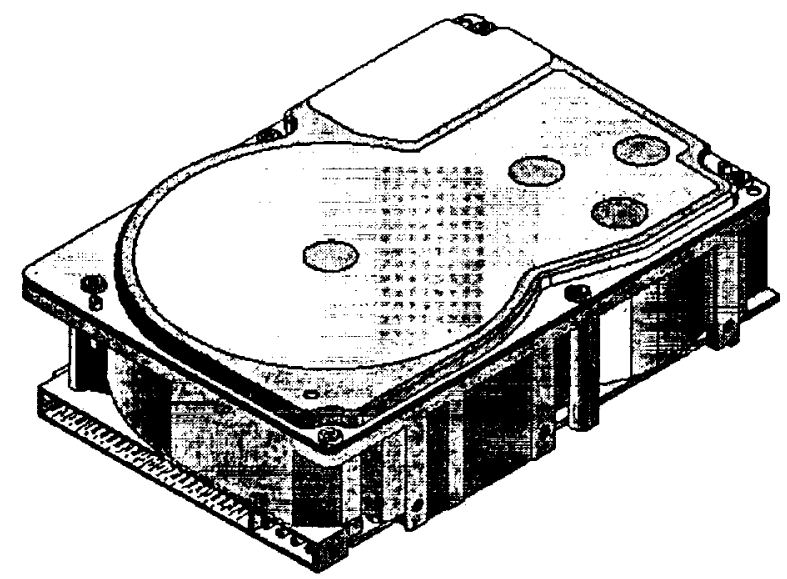

Figure 3. Seagate Barracuda 18

At the time, the Seagate Barracuda 18.2GB disk drive (shown above in Figure 3) was the largest disk drive available with a manual that contained supporting data that could help aid in achieving some of the study objectives. After comparing the data of the Barracuda disk drive with other tape and disk drives, it was determined to be the best-suited drive to meet the requirements of SPARCLE with some modifications.

The MSFC Computer and Data Systems Group agreed to provide and deliver to the SPARCLE program a MSU with the capacity of 90GB that could be packaged in a rugged chassis designed to meet the size and weight requirements. This would require a chassis design with wedgelock technology capability to house and lock the five disk drives into designed slots in the walls of the inner chassis. To meet the MSU requirements of at least $142 \mathrm{~GB}$ of data storage, with a MSU that only provides $90 \mathrm{~GB}$, the Digital Systems and Software Development teams agreed to provide algorithms to compress the data. The data would be compressed to ratio of at least $2: 1$, before storing the data into packets for the downlink telemetry stream. The control interface between the CPU card and the MSU is a SCSI II that links all five disk drives in parallel.

The first design approach involved the design of a inner chassis that could house the five disk drives with vibration isolators that were rated to meet the requirements of Hitchhiker. The isolators were selected based on the amount of weight that they support to damp out the vibrations that the disk drive would experience. The total weight of the inner chassis was calculated to be 17.22 lbs., where each disk drive weighed $2.2 \mathrm{lbs}$.

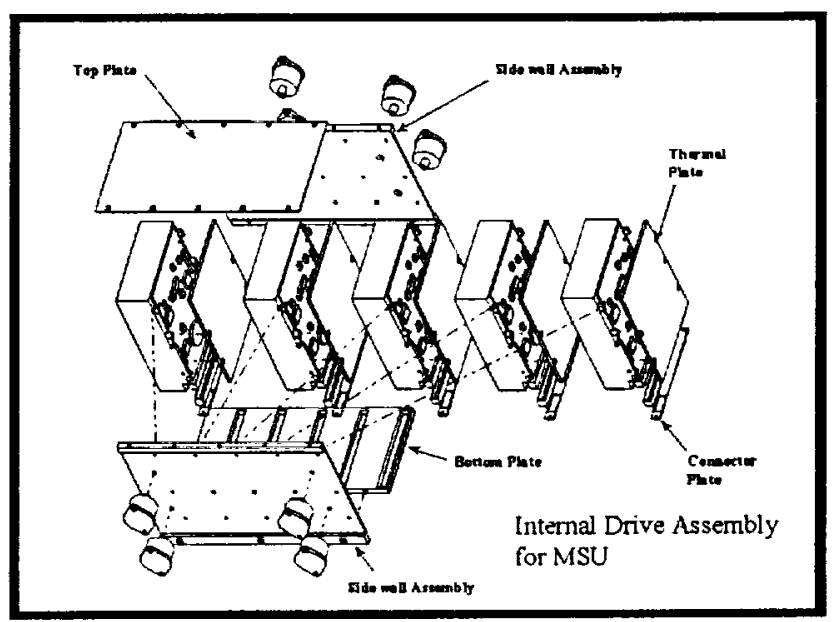

Figure 4. MSU Inner Chassis with Isolators

The selected isolators were required to be rated at 3 lbs. because total number of isolator times their rating would equal the maximum weight allowed to be supported and to function per specification. However, the problem with the design isolating the disk drives from vibrations is that the heat is also isolated. Therefore, the design of the chassis had to include designs that could incorporate a method to carry the heat from the inner chassis to the outer chassis' baseplate using conduction.

\section{Testing To Meet Qualifications}

The mass storage devices were tested in all three axes to a level $-6 \mathrm{db},-3 \mathrm{db}$, and $0 \mathrm{db}$ from 
the Hitchhiker requirements. Also, one disk drive was tested in all three axes to a level $3 \mathrm{db}$ above the requirement. After it had been fully determined that the disk drives could survive the required levels of vibrations, another study was conducted to determine the best approach for resolving the disk drives' thermal worst case scenario while operating under the SPARCLE program's thermal requirements. Therefore, several thermal tests were conducted to determine how effective the heat could be pulled away from the walls of the disk drives using methods of conduction. The concept of using flexible grounding straps was explored and tested (as shown in Figure 5 below). Testing determined that the braided wire of the flexible grounding strap had to many cross braid thermal junctions that produced a thermal resistance to great to remove the required amount of heat.

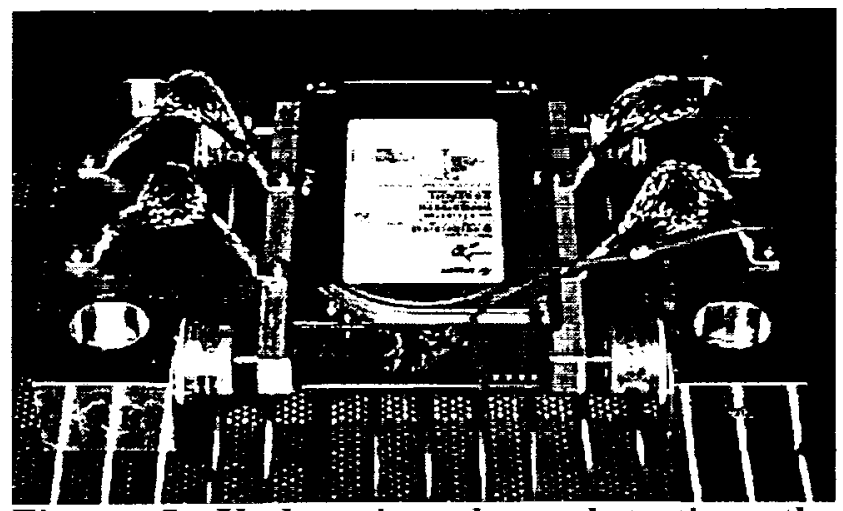

Figure 5. Undergoing thermal testing, the Seagate disk drive is configured utilizing four flexible ground straps to conduct heat away from the side rails mounted to the walls drive.

Therefore, this method could not effectively remove the heat to achieve the required results needed to reduce the thermal build-up on each drive in their operating conditions.

Several components on the disk drive's printed circuit board were profiled and determined that the heat produced must be removed directly from the component to control over heating. Initially, tests were conducted to verify that the heat could be removed from the disk drives' PCB by using metal fingers in

contact with the components on the PCB. With the metal fingers attached to a thermal plate, as shown in Figure 6 below, the heat could be drawn out and away from the disk drives' components. Testing determined that the heat could be drawn from the components effectively, if the thermal junctions could be reduced as close as possible to zero resistance at every critical point between the disk drives' components and the base plate.

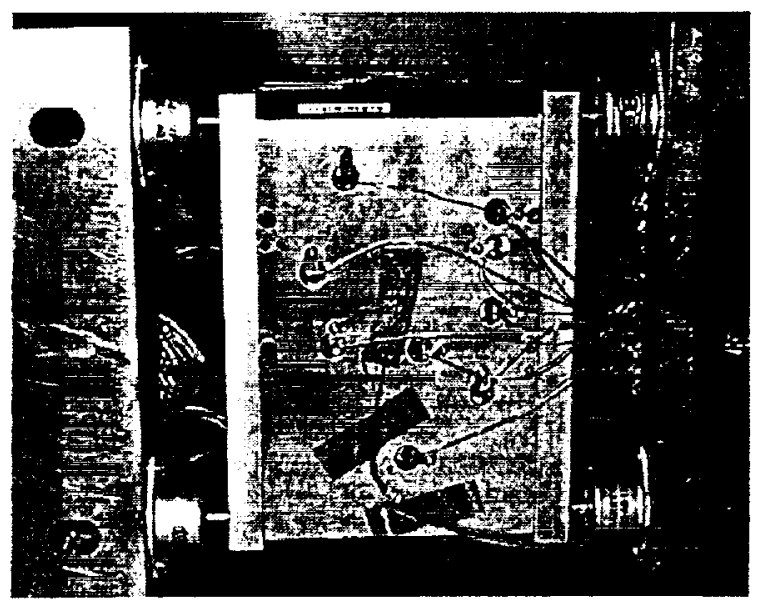

Figure 6. Disk Drive in thermal chamber with thermocouples attached to each metal finger.

This would require more effective materials to achieve this objective because the drives failed at $53^{\circ} \mathrm{C}$. just 3 degrees above the required qualification level. The burned interface on the disk drives shown below in Figure 7 is the interface to the internal motor controller on the disk drives' PCB.

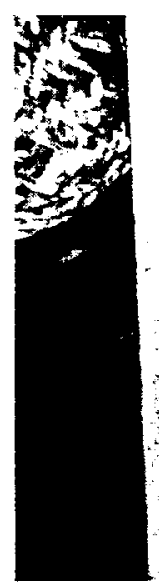

Figure 7.

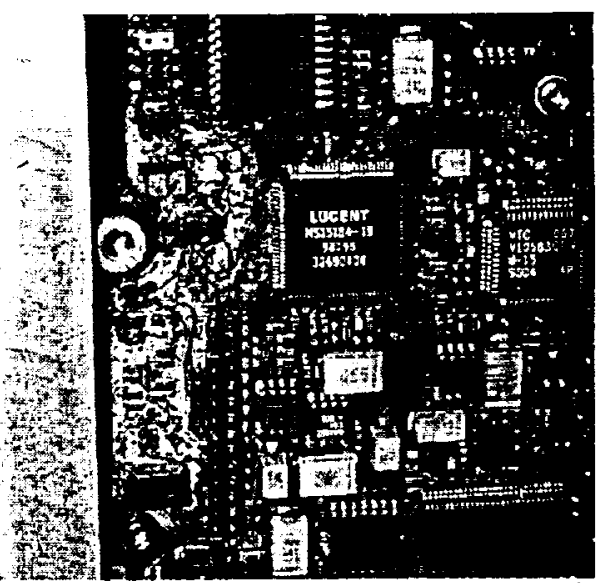
Disk drive burned at interface 
The disk drives' thermal characteristics as given by the manufacture drove the requirements to use software to manage the power delivered to each drive and to control the drive modes. Switching them into a low-powerconsumption mode would help to reduce the thermal build-up within the MSU. The power to each disk drive is controlled and delivered by the Power Distribution Unit (PDU). A 5-bit discrete command signal from the CDMS to the PDU will select and control the disk drive to be used. The first two bits will control the "power on" and the "spin up" modes to the disk drives. The last three bits will be used to select which disk drive to control. With this methodology of control, there will never be more than two drives on at any given time, as designed by the software. Therefore, the search began to find materials to remove the heat from the disk drives' components and to transfer it to a thermal plate and the baseplate.

\section{Final Design}

A high thermally conductive gap filling material (shown in Figure 8) was found and designed into this application. The gap pads are made from a highly conformable low modulus polymer that acts as a thermal interface between electrical components and heat sinks.

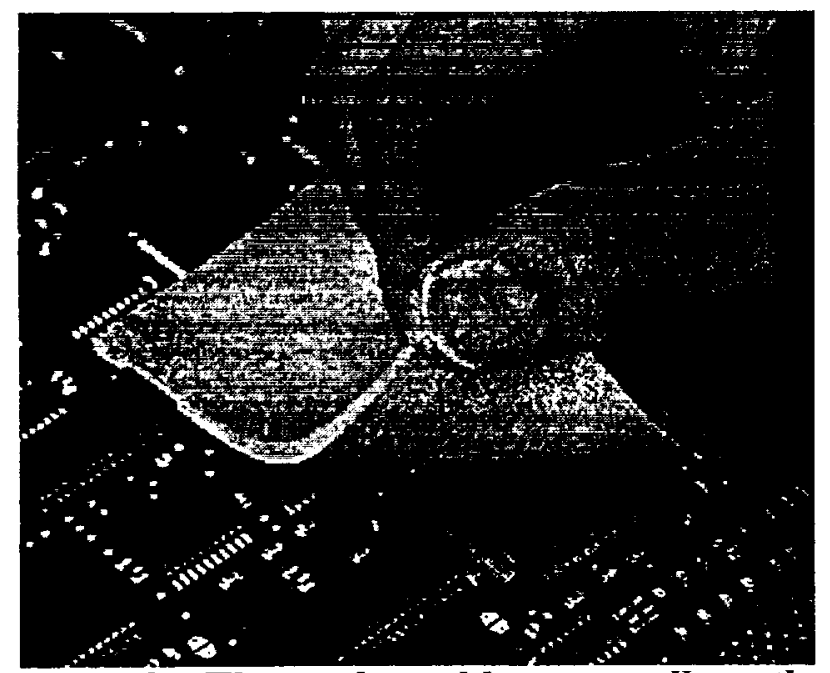

Figure 8. The conformable nature allows the material to fill air gaps to enhance the thermal performance of electrical systems.
Now that the heat could be removed from the disk drive PCB components, it needed to be transferred to the baseplate of the MSU chassis. Since copper has some of the best thermal conductivity properties, a $2 \times 8$ inch flex-rigid assembly was designed (as shown in figure 9) using 8 layers of 4 ounces copper, to transfer the heat from the thermal plate to the baseplate.

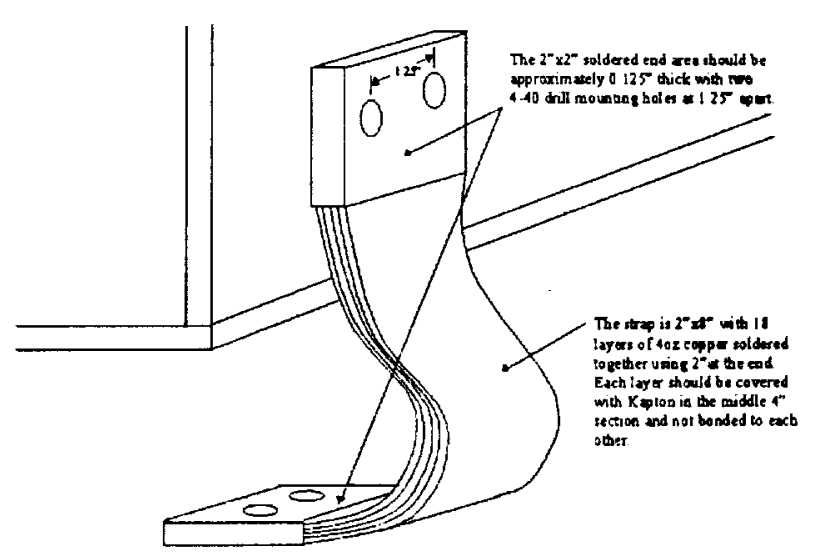

Figure 9. The thermal strap concept design.

This concept is still being tested, but some of the results thus far have proven to be favorable.

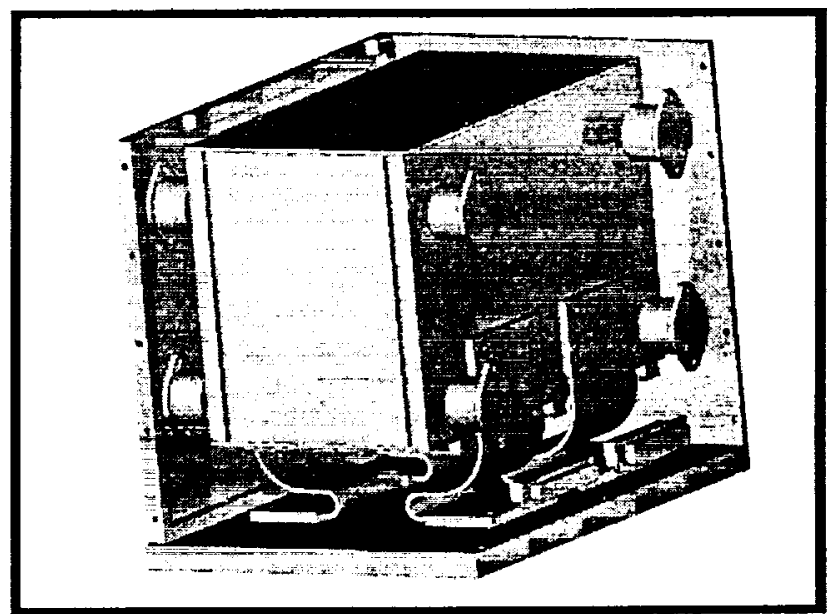

Figure 10. The 3D drawings of the thermal straps show how they are to be designed into the Final MSU Chassis concept.

It was determined that some type of thermal pad should be used between each metal junction of the thermal straps with the walls of the MSU inner chassis and the outer chassis's baseplate to increase the thermal performance. 


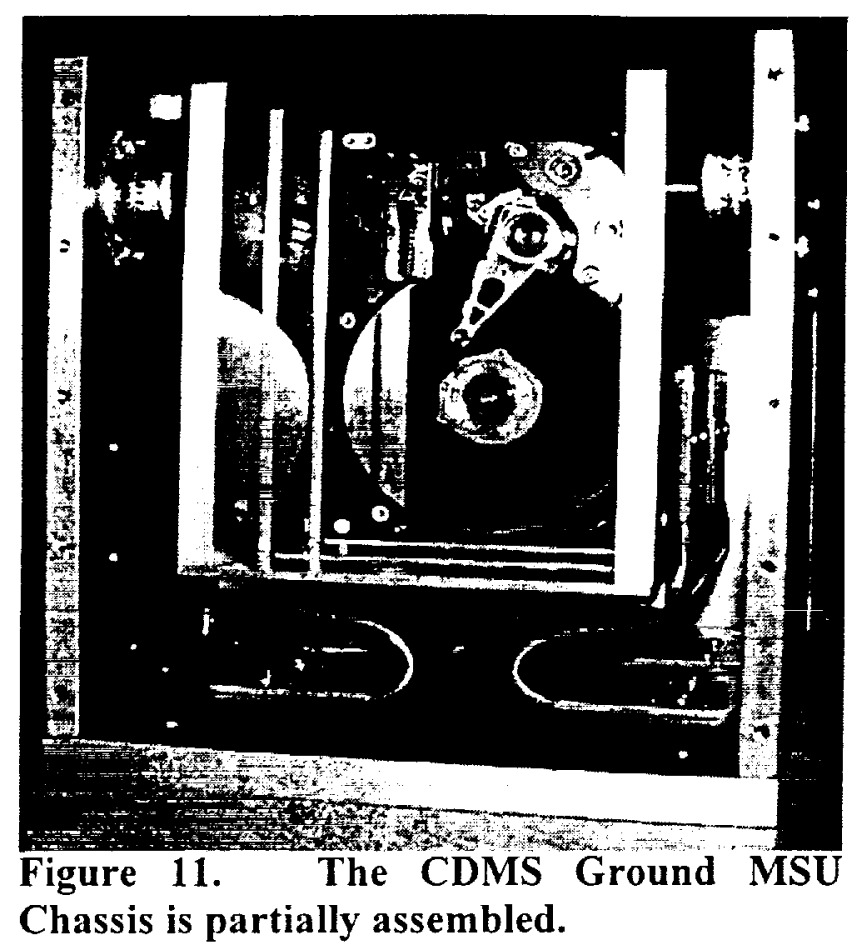

\section{Lessons Learned}

The final ground MSU must still undergo the final qualification testing before the concept can be proven to be successful. It is understood that COTS products may have some unpredictable design margins and manufacturing practices in spite of their characteristics from a long line of massproduced lots. To verify that the COTS product could meet the requirements of the program, early environmental and performance testing had to be conducted on sample devices to verify that the vendors' product was in compliance with the specifications given in the vendors manual. This type of testing allowed the unknown characteristics of the selected devices to be characterized and measured. Thus, the characteristics could be added in the design modifications needed to aid the COTS in meeting the program requirements.

It was determined that the design margins became a critical point in the modifications of the chassis design. Due to the thermal failure, it appeared that the COTS product would have to operate near the boundaries of it performance and environmental envelopes. Therefore, more time with the modified version of the COTS product is required to generate additional data over to design in additional safety margins. Reverse engineering could become necessary to acquire the data if adequate vendor information is not obtained.

Testing these products to their maximum limits without the modification proved only to be helpful in verifying that they could not survive testing much beyond their specified environment. It was also determined that streamlining the fabrication process could prove to be very helpful before any hardware is to be modified. Using the Stereolithography (STL) rapid prototyping process can prove its usefulness in the discovery of problems undetected within the $3 \mathrm{D}$ computer aided design (CAD) tools. This process could prove to reduce the manufacturing process, if fully implemented in the fabrication phase when cast aluminum chassis are built directly from the STL files generated by the CAD program. To produce an error free piece of hardware from the first time cut with the manufacture, several iterations to produce an error free STL model may be required. However, this methodology will allow the ability to exercise and implement a practice to develop systems for any given program, "...faster, cheaper, and better..." 